\title{
Article \\ Symmetry Analysis of Magnetoelectric Effects in Perovskite-Based Multiferroics
}

\author{
Zukhra Gareeva ${ }^{1,2, * \mathbb{D}}$, Anatoly Zvezdin ${ }^{3}$, Konstantin Zvezdin ${ }^{3}$ and Xiangming Chen ${ }^{4}$ \\ 1 Institute of Molecule and Crystal Physics, Subdivision of the Ufa Federal Research Centre of the Russian \\ Academy of Sciences, Prospect Octyabrya 151, 450075 Ufa, Russia \\ 2 Institute of Physics and Technology, Bashkir State University, ul. Z. Validi 32, 450076 Ufa, Russia \\ 3 Prokhorov General Physics Institute of the Russian Academy of Sciences, ul. Vavilova 38, \\ 119991 Moscow, Russia; zvezdin.ak@phystech.edu (A.Z.); zvezdin.ka@phystech.edu (K.Z.) \\ 4 Laboratory of Dielectric Materials, School of Materials Science and Engineering, Zhejiang University, \\ Zheda Road 38, Hangzhou 310027, China; xmchen59@zju.edu.cn \\ * Correspondence: zukhragzv@yandex.ru; Tel.: +7-(917)-8049560
}

check for updates

Citation: Gareeva, Z.; Zvezdin, A.; Zvezdin, K.; Chen, X. Symmetry Analysis of Magnetoelectric Effects in Perovskite-Based Multiferroics. Materials 2022, 15, 574. https:// doi.org/10.3390/ma15020574

Academic Editors: Evgeny Parfenov and Elena Korznikova

Received: 13 December 2021

Accepted: 7 January 2022

Published: 13 January 2022

Publisher's Note: MDPI stays neutral with regard to jurisdictional claims in published maps and institutional affiliations.

Copyright: (C) 2022 by the authors. Licensee MDPI, Basel, Switzerland. This article is an open access article distributed under the terms and conditions of the Creative Commons Attribution (CC BY) license (https:// creativecommons.org/licenses/by/ $4.0 /)$.

\begin{abstract}
In this article, we performed symmetry analysis of perovskite-based multiferroics: bismuth ferrite $\left(\mathrm{BiFeO}_{3}\right)$-like, orthochromites $\left(\mathrm{RCrO}_{3}\right)$, and Ruddlesden-Popper perovskites $\left(\mathrm{Ca}_{3} \mathrm{Mn}_{2} \mathrm{O}_{7}\right.$-like), being the typical representatives of multiferroics of the trigonal, orthorhombic, and tetragonal crystal families, and we explored the effect of crystallographic distortions on magnetoelectric properties. We determined the principal order parameters for each of the considered structures and obtained their invariant combinations consistent with the particular symmetry. This approach allowed us to analyze the features of the magnetoelectric effect observed during structural phase transitions in $\mathrm{Bi}_{x} \mathrm{R}_{1-x} \mathrm{FeO}_{3}$ compounds and to show that the rare-earth sublattice has an impact on the linear magnetoelectric effect allowed by the symmetry of the new structure. It was shown that the magnetoelectric properties of orthochromites are attributed to the couplings between the magnetic and electric dipole moments arising near $\mathrm{Cr}^{3+}$ ions due to distortions linked with rotations and deformations of the $\mathrm{CrO}_{6}$ octahedra. For the first time, such a symmetry consideration was implemented in the analysis of the RuddlesdenPopper structures, which demonstrates the possibility of realizing the magnetoelectric effect in the Ruddlesden-Popper phases containing magnetically active cations, and allows the estimation of the conditions required for its optimization.
\end{abstract}

Keywords: keyword multiferroics; crystal lattices; symmetry; perovskites; magnetoelectric effect

\section{Introduction}

Multiferroic, multifunctional materials attract enormous research activity due to the prospects of their implementation in science and technology. The collective state parameters attainable in multiferroics (MFs) are preferable for advanced spintronic devices based on the use of combinatorial functions due to their high efficiency, low power consumption, and adaptability [1].

Despite the variety of currently known magnetoelectric materials, perovskite-like structures remain the most researched MFs compounds. The family of perovskites stands out for its diversity due to the intrinsic instability of the cubic parent perovskite phase [2]. Crystallographic distortions owing to the substitution of various cations at A and B positions can lead to the emergence of ferroelectric and MF properties due to the hybridization of ion orbitals, tilt of oxygen octahedra $\left(\mathrm{BO}_{6}\right)$, doping of various cations, and other factors [3]. $\mathrm{ABO}_{3}$ perovskites can produce a variety of MFs differing in the type of (i) ferroelectric ordering (A- or B-driven ferroelectricity), as in the cases of $\mathrm{EuTiO}_{3}$ [4] and $\mathrm{BiFeO}_{3}$ [5]; (ii) magnetic ordering (although most perovskite MFs are G-type antiferromagnets (AFM), canted ferromagnetism and a combination of ferromagnetic and AFM phases are also possible) [6,7]; (iii) mechanism of magnetoelectric coupling, which can be associated with 
magnetoelectrically active d-ions or f-ions $\left(\mathrm{RFeO}_{3}\right)$ [8], the Dzyaloshinskii-Moriya interaction $\left(\mathrm{BiFeO}_{3}\right)$ [5], spin-orbit coupling $(\mathrm{BiMnTe})$ [9], antiferroelectric orderings $\left.\left(\mathrm{RCrO}_{3}\right)\right)$ [10]; and (iv) temperatures of ferroelectric and magnetic orderings, which remain low enough for most perovskite-based MFs.

Quite recently, the MF community turned to hybrid layered perovskite structures, considering them as prospective candidates for high-temperature MFs. We refer to layered structures composed of an n-layered perovskite with a single-layer spacer in-between. Depending on the kind of single-layer spacer, one can distinguish the Ruddlesden-Popper (RP) $\mathrm{A}_{2}^{\prime}{ }_{2}\left(\mathrm{~A}_{\mathrm{n}-1} \mathrm{~B}_{\mathrm{n}} \mathrm{O}_{3 \mathrm{n}+1}\right)$, Dion-Jacobson (DJ) $\mathrm{A}^{\prime}\left(\mathrm{A}_{\mathrm{n}-1} \mathrm{~B}_{\mathrm{n}} \mathrm{O}_{3 \mathrm{n}+1}\right)$, and Aurivillius $\mathrm{Bi}_{2} \mathrm{O}_{2}\left(\mathrm{~A}_{\mathrm{n}-1} \mathrm{~B}_{\mathrm{n}} \mathrm{O}_{3 \mathrm{n}+1}\right)$ structures $[11,12]$. When $n=\infty$, the layered perovskite transforms into a classical perovskite.

Among layered perovskites, the most widespread and studied compounds are the RP structures. They received their name in honor of the chemists R. Ruddlesden and P. Popper, who, in 1957, synthesized the series of $\mathrm{Sr}_{2} \mathrm{TiO}_{4}, \mathrm{Sr}_{3} \mathrm{Ti}_{2} \mathrm{O}_{7}$, and $\mathrm{Sr}_{4} \mathrm{Ti}_{3} \mathrm{O}_{10}$ phases, forming a class of complex oxides $\mathrm{S}_{\mathrm{rn+1}} \mathrm{Ti}_{n} \mathrm{O}_{3 n+1}$ [13]. Since then, the class of RP structures has expanded significantly, and, at present, it includes various compounds with the general formula $\mathrm{A}_{2}^{\prime}\left(\mathrm{A}_{\mathrm{n}-1} \mathrm{~B}_{\mathrm{n}} \mathrm{O}_{3 \mathrm{n}+1}\right)$, where $\mathrm{A}^{\prime}=\mathrm{La}, \mathrm{Sr}, \mathrm{Li}, \ldots ; \mathrm{A}=\mathrm{Sr}, \mathrm{Ca}, \mathrm{K}, \ldots$; and $\mathrm{B}=\mathrm{Ti}, \mathrm{Fe}, \mathrm{Mn}$, $\mathrm{Nb}, \ldots$. The advantages of RP-type perovskites are related to long-term chemical stability, the activity of oxygen reduction reaction, and the possibility of realizing nontrivial magnetic and electrical properties [11-17]. Due to the activity of the reaction with oxygen, RP structures find applications in solid oxide fuel cells [14]. As for magnetoelectricity, interest in layered perovskites is associated with the discoveries of the colossal magnetoresistance effect in the early 2000s in RP structures with magnetoactive cations $\left(\mathrm{Ca}_{3} \mathrm{Mn}_{2} \mathrm{O}_{7}, \ldots\right)$ [14-19] and ferroelectricity at room temperatures, found in $\mathrm{Ca}_{3-x} \mathrm{Sr}_{x} \mathrm{Ti}_{2} \mathrm{O}_{7}$ in 2015 [20] and then in $\mathrm{Sr}_{3} \mathrm{M}_{2} \mathrm{O}_{7}$-based oxides and other RP phases [21]. Currently, RP structures are considered promising candidates for high-temperature MFs. The crystal structure, instabilities, and structural phase transitions occurring in RP oxides are well known; however, a number of questions concerning ferroelectricity, magnetic ordering, and their coupling is open for discussion.

The focus of this manuscript was the study of the magnetoelectric effect in $\mathrm{Bi}_{1-x} \mathrm{R}_{\mathrm{x}} \mathrm{FeO}_{3}$, $\mathrm{RCrO}_{3}$, and $\mathrm{Ca}_{3}\left(\mathrm{Ti}_{1-\mathrm{x}} \mathrm{Mn}_{\mathrm{x}}\right)_{2} \mathrm{O}_{7}$ multiferroics (MFs). We aimed to develop a unified approach to the analysis of the perovskite-like multiferroics based on the symmetry of the material, taking crystallographic distortions as the primary order parameters.

It is valuable to note that the classification of distortions and the search for recipes for effective magnetoelectric couplings in MFs with a perovskite structure is a long-standing and, at the same time, 'hot' problem. We refer to several reviews, references therein, and original papers that discussed various classification schemes for perovskite distortions and group-theoretical approaches, including their implementation in online tools (in particular, ones on the Bilbao Crystallographic Server) for identification of active order parameters, and their possible couplings and invariant polynomials [22-29]. However, distortion classification schemes mainly deal with $\mathrm{BO} 6$ octahedrons and do not account for polar distortions, which have significant impact on ferroelectric and MF properties; in addition, they also have limitations for specific systems. Despite the power of the software tools, they have not yet been applied to each MF system; for example, the details of the magnetoelectric coupling in MFs with a variable concentration of rare-earth ions have not yet been investigated, and the same statement is also applied to the RP structures.

Thus, in our research, we appealed to group-theoretical analysis, which is an elegant and effective tool for studying the properties of crystals with a complex magnetic structure, to classify the distortive, ferroelectric, and magnetic orderings in several classes of perovskite MFs. For this purpose, we examined typical MF structures with the perovskite parent phase: $\mathrm{BiFeO}_{3}, \mathrm{RCrO}_{3}, \mathrm{Ca}_{3} \mathrm{Mn}_{2} \mathrm{O}_{7}$, in which small distortions in the crystallographic lattice lead to systems of different symmetry (trigonal, orthorhombic, and tetragonal). Using the methods of theoretical group analysis, we considered how the symmetry manifests itself in their magnetoelectric properties. We determined magnetic and structural modes, 
classified them according to the irreducible representations of the corresponding symmetry group, and calculated the coefficients of magnetoelectric couplings.

\section{Materials and Methods}

In this section, we analyze the crystal structure, and ferroelectric and magnetic properties of representatives of the perovskite-based multiferroics and, using the methods of the theoretical group analysis, determine the features of their magnetoelectric properties.

\subsection{Multiferroic $\mathrm{BiFeO}_{3}$}

We start our consideration with the well-known multiferroic $\mathrm{BiFeO}_{3}$ with high temperatures of ferroelectric and magnetic orderings $\left(\mathrm{T}_{\mathrm{C}}=1083 \mathrm{~K}, \mathrm{~T}_{\mathrm{N}}=643 \mathrm{~K}\right) . \mathrm{BiFeO}_{3}$ crystallizes in the nonpolar symmetry group $R 3 c$ and is a ferroelectric material. The high-symmetry perovskite group is reduced to $R 3 c$ due to three types of distortions: (i) relative displacement of $\mathrm{Bi}$ and $\mathrm{Fe}$ ions along <111> axis, (ii) deformations of oxygen octahedrons, and (iii) counterrotation of oxygen octahedrons around Fe ions. The displacements of $\mathrm{Bi}$ and $\mathrm{Fe}$ ions from their centrosymmetric positions lead to spontaneous electric polarization $\boldsymbol{P}_{\boldsymbol{s}}$ directed along one of the $<111>$ crystallographic axes [30]. The magnitude of polarization has been the subject of controversy for a while. As was reported in Refs. [5,20,31-35], spontaneous polarization in the $\mathrm{BiFeO}_{3}$ crystal and films can attain values varying from 6 to $150 \mu \mathrm{C} / \mathrm{cm}^{2}$. Electric polarization is sufficiently low, $P_{s} \sim 6-9 \mu \mathrm{C} / \mathrm{cm}^{2}$, in single crystals and ceramic samples [34,35]; the large values of $\boldsymbol{P}_{\boldsymbol{s}}$, around $100 \mu \mathrm{C} / \mathrm{cm}^{2}$, are achieved under epitaxial strain in the films [5,36-38]; and they can also be related to the supertetragonal phase induced by the strain.

The rigorous estimation of electronic polarization requires the implementation of quantum mechanics, including electronic structure methods with correspondence to experimentally measurable observables [39]. In the frame of these approaches in Ref. [40], electric polarization in perovskite $\mathrm{BiFeO}_{3}$ was calculated as a function of percentage distortion from the high-symmetry nonpolar structure to the ground-state $R 3 c$ structure, which gives the value $95.0 \mu \mathrm{C} \mathrm{cm}^{-2}$. However, as was shown in Ref. [30], similar results can be obtained with the use of the point charge model, where the electric polarization is represented through the atomic displacements of $\mathrm{Bi}^{3+}$ and $\mathrm{O}^{2-}$ ions.

$$
P=\frac{4 e}{V}\left(3 \zeta_{R}-4 \zeta_{O 1}-2 \zeta_{O 3}\right)
$$

where $e$ is the elementary charge, $V$ is the unit cell volume, $\zeta_{R}$ is the displacement of Bi ions corresponding to (i) distortion, and $\zeta_{\mathrm{O} 1}$ and $\zeta_{\mathrm{O} 3}$ are the displacements of oxygen ions corresponding to (ii)-distortion.

In magnetic relation, $\mathrm{BiFeO}_{3}$ is weak ferromagnet with G-type antiferromagnetic (AFM) ordering characterized by AFM vector $L=\frac{1}{V} \sum_{i=1}^{6}(-1)^{i} \mu_{i}$, where $\mu_{i}$ are the magnetic moments of six Fe ions in a unit cell. The canting of spins is related to rotations of oxygen octahedrons FeO6, determined by the vector $\Omega$, the distortions of (iii) - type [41], which have an impact on the weak ferromagnetic vector, determined as

$$
\boldsymbol{M}=\frac{V_{0} a^{2}}{6 J} \boldsymbol{\Omega} \times \boldsymbol{L}+\frac{V_{0} a^{2}}{6 J} \sum_{n=1}^{6}\left(\boldsymbol{n}_{n} \times \zeta_{O_{n}}\right)
$$

where $V_{0}$ is a constant, $a$ is the lattice parameter, $J$ is the exchange constant, and $\boldsymbol{n}_{n}$ is the direction vector, oriented along one of the $<100>$ crystallographic axes. Here, we used an assumption that the Heisenberg exchange constant remains unchanged, which is a commonly used approximation for multiferroic $\mathrm{BiFeO}_{3}$; in particular, the deviation (noncollinearity) of the nearest-neighbor spins is accounted for by the antisymmetric Dzyaloshinskii-Moriya exchange interaction [42,43]. AFM spin arrangement is superimposed with a spatially modulated magnetic structure with a large period $\lambda=(620 \pm 20 \AA)$ incommensurate with 
the lattice parameter [44]. The magnetic moments of Fe ions retain their local mutually antiferromagnetic G-type orientation and rotate along the propagation direction of the modulation in the plane perpendicular to the basal crystal plane.

To explore the magnetoelectric effect (MEE) in $\mathrm{BiFeO}_{3}$, we appealed to symmetry analysis. Knowledge of crystal structure and symmetry is of primary importance for this approach. To consider the properties of $\mathrm{BiFeO}_{3}$, the space group $R 3 \bar{c}$ was taken as a 'parent' symmetry phase [45]. $R 3 \bar{c}$ differs from the space symmetry group $R 3 c$ of $\mathrm{BiFeO}_{3}$ single crystals only by the presence of a polar vector $\boldsymbol{P}=\left(0,0, P_{s}\right)$. The symmetry analysis of the magnetoelectric properties of $\mathrm{BiFeO}_{3}$ has been performed in Refs. [45-47]. Here, we focused our attention on the magnetoelectric tensor $\alpha_{i j}$, which links the electric polarization and the magnetic field

$$
P_{i}=\alpha_{i j} H_{j}
$$

As was shown in Ref. [47] for $\mathrm{BiFeO}_{3}$ crystals, the tensor $\alpha_{i j}$ can be expressed through the components of the AFM vector $L$

$$
\alpha_{i j}^{\mathrm{BiFeO}_{3}}=\left|\begin{array}{lrr}
-a_{1} L_{x} & a_{4} L_{z}+a_{1} L_{y} & a_{2} L_{y} \\
a_{1} L_{y}-a_{4} L_{z} & a_{1} L_{x} & -a_{2} L_{x} \\
-a_{3} L_{y} & a_{3} L_{x} & 0
\end{array}\right|
$$

As seen from Equations (3) and (4), the symmetry allows the linear magnetoelectric effect; however, due to the presence of an incommensurate space-modulated structure, it is not observed in $\mathrm{BiFeO}_{3}$. Thus, when the spin cycloidal structure is destroyed, the linear magnetoelectric effect is restored. It can be achieved by applying a strong magnetic field, pressure, or doping $\mathrm{BiFeO}_{3}$ with rare-earth ions. In recent years, a series of experimental studies has been conducted, indicating the enhancement of the magnetoelectric effect in $\mathrm{BiFeO}_{3}$ multilayers and composites.

Here, we discuss the replacement of various rare-earth $(\mathrm{RE})$ ions at the A-positions as a way to improve the MF properties of $\mathrm{BiFeO}_{3}$, a route that was paved in the 1990s [30,45-50], and current research confirms its efficiency [6,51]. As was shown in Ref. [51], La-substitution in $\mathrm{Bi}_{1-\mathrm{x}} \mathrm{La}_{\mathrm{x}} \mathrm{FeO}_{3}(\mathrm{x}=0.08 \sim 0.22)$ ceramics induces a continuous structural evolution from $R 3 c$ to $P n a 2_{1}$ and, finally, the Pbnm phase. To understand the properties of $\mathrm{Bi}_{1-\mathrm{x}} \mathrm{R}_{\mathrm{x}} \mathrm{FeO}_{3}$ structures $(\mathrm{R}=\mathrm{La}, \mathrm{Gd}, \mathrm{Dy}, \ldots)$, it is reasonable to introduce the AFM vector $(l)$, which characterizes the magnetic ordering of the rare-earth sublattice, and to consider the Pbnm space symmetry group as the parent phase in the $\mathrm{Bi}_{1-x} \mathrm{R}_{\mathrm{x}} \mathrm{FeO}_{3}$ structure, keeping in mind structural phase transitions $\mathrm{Pbnm} \rightarrow \mathrm{Pna}_{2}$. The space group Pbnm contains eight irreducible representations, where their matrix representations are given in the columns corresponding to the generating symmetry elements (Table 1 ). The vector components of magnetic field $\boldsymbol{H}$, electric polarization $\boldsymbol{P}$, magnetization $\boldsymbol{M}$, and antiferromagnetic vectors $\boldsymbol{L}$ and $\boldsymbol{l}$ form the irreducible representations and are placed in the last column in Table 1 according to their transformation properties. Table 1 allows the determination of the transformation properties of AFM vector components, exchange-coupled structures, and magnetoelectric invariants, contributing to the magnetoelectric energy of the $\mathrm{Bi}_{1-x} \mathrm{R}_{\mathrm{x}} \mathrm{FeO}_{3}$ structure.

$$
\begin{gathered}
\Phi_{m e}^{\mathrm{B}_{1-x} \mathrm{R}_{x} \mathrm{FeO}_{3}}=P_{z}\left(\gamma_{1}\left(l_{z} M_{y}-l_{y} M_{z}\right)+\gamma_{2}\left(l_{x} L_{y}-l_{y} L_{x}\right)\right)+P_{x}\left(\gamma_{x y x}\left(l_{y} M_{x}-l_{x} M_{y}\right)+\gamma_{2}\left(l_{y} L_{z}-l_{z} L_{y}\right)\right)+ \\
P_{y}\left(\gamma_{3} l_{x} M_{x}+\gamma_{2} l_{x} L_{z}+\gamma_{4}\left(l_{y} M_{y}+L_{y}^{2}\right)\right)
\end{gathered}
$$

where $\gamma_{i}$ are the magnetoelectric coefficients,

$$
\gamma_{1}=\gamma_{z z y}, \gamma_{2}=\gamma_{x y z}, \gamma_{3}=\gamma_{y x x}, \gamma_{4}=\gamma_{y y y}
$$


Table 1. Irreducible representations of the Pbnm symmetry group.

\begin{tabular}{cccccccc}
\hline$\Gamma_{\boldsymbol{i}}$ & $\overline{\mathbf{1}}$ & $\mathbf{2}_{\mathbf{x}}$ & $\mathbf{2}_{\mathbf{y}}$ & $\mathbf{2}_{\mathbf{z}}$ & Order Parameters, and Magnetic and Electric Fields \\
\hline$\Gamma_{1}$ & 1 & 1 & 1 & 1 & $L_{y}$ & & $H_{x}$ \\
\hline$\Gamma_{2}$ & 1 & 1 & -1 & -1 & $M_{x}$ & $L_{y}$ \\
\hline$\Gamma_{3}$ & 1 & -1 & 1 & -1 & $M_{y}$ & $L_{x}$ & \\
\hline$\Gamma_{4}$ & 1 & -1 & -1 & 1 & $M_{z}$ & $H_{z}$ \\
\hline$\Gamma_{5}$ & -1 & 1 & 1 & 1 & $l_{y}$ & $P_{z}$ & $E_{y}$ \\
\hline$\Gamma_{6}$ & -1 & 1 & -1 & -1 & $l_{x}$ & $P_{y}$ & $E_{x}$ \\
\hline$\Gamma_{7}$ & -1 & -1 & 1 & -1 & & $P_{x}$ & \\
\hline$\Gamma_{8}$ & -1 & -1 & -1 & 1 & $l_{z}$ & & \\
\hline
\end{tabular}

The magnetoelectric coupling coefficient for the $\mathrm{Bi}_{1-\mathrm{x}} \mathrm{R}_{\mathrm{x}} \mathrm{FeO}_{3}$ structures can be determined as follows

$$
\alpha_{i j}=\left|\begin{array}{ccc}
a_{1}\left(l_{x} L_{x}+l_{z} L_{z}\right) & a_{12} l_{x} & -a_{13} l_{y} \\
a_{12} l_{z} & a_{2} l_{y} & a_{23} l_{x} \\
a_{13} l_{y} & a_{32} l_{z} & a_{1}\left(l_{x} L_{x}+l_{z} L_{z}\right)
\end{array}\right| \approx\left|\begin{array}{ccc}
0 & a_{12} l_{x} & -a_{13} l_{y} \\
a_{12} l_{z} & a_{2} l_{y} & a_{23} l_{x} \\
a_{13} l_{y} & a_{32} l_{z} & 0
\end{array}\right|
$$

In the case of $\mathrm{Bi}_{1-x} \mathrm{R}_{\mathrm{x}} \mathrm{FeO}_{3}$, rare-earth elements can have a significant impact on magnetoelectric properties. As seen from Equation (6), $\alpha_{i j}$ depends on the components of the AFM vector of the rare-earth sublattice (for the case of nonzero magnetization on the $R$ ion site), and it is possible to distinguish a linear MEE related to rare-earth elements, and a quadratic MEE emerges due to cross-coupling of the AFM vectors of iron and rare-earth sublattices.

\subsection{Magnetoelectric Properties of $\mathrm{RCrO}_{3}$}

In this section, we consider MEE in the rare-earth orthochromites $\left(\mathrm{RCrO}_{3}\right)$, focusing on the relation of magnetoelectric properties with crystallographic distortions. The crystal structure of $\mathrm{RCrO}_{3}$ belongs to the space symmetry group Pbnm/Pnma [52-54]. Its orthorombically distorted perovskite unit cell contains $4 \mathrm{RCrO}_{3}$ molecules (Figure 1). In addition, $4 \mathrm{Cr}^{3+}$ and $4 \mathrm{R}^{3+}$ magnetic ions (in the case of $\mathrm{R}=$ rare-earth ion) are located in the local positions differing by the symmetry of $\mathrm{O}^{2-}$ environments. The $d$-ions $\left(\mathrm{M}^{3+}\right)$ occupy the position $4 b$, the $f$-ions $\left(\mathrm{R}^{3+}\right)$ occupy the position $4 c$, and oxygen ions occupy the positions $4 c$ and $8 d$ (in Wyckoff notation).

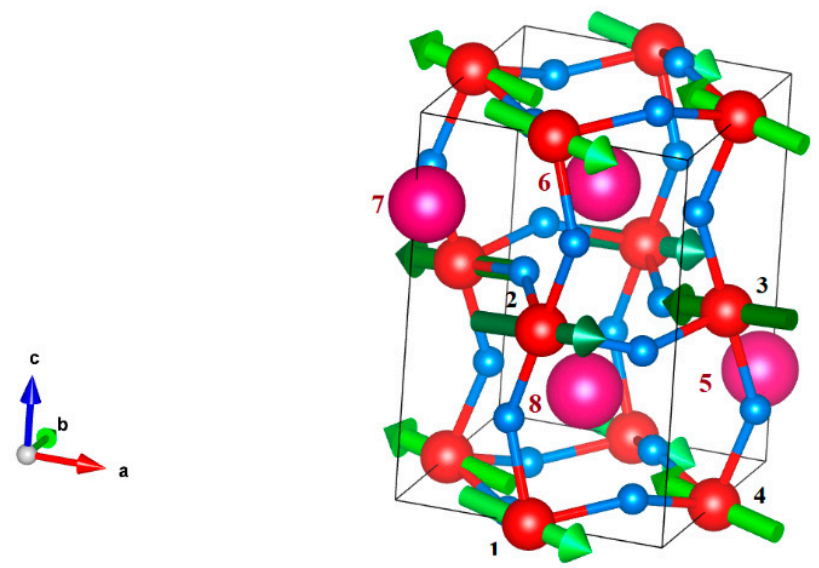

Figure 1. Electric dipole moments arrangement in $\mathrm{RCrO}_{3}$ unit cell, a, b, c are the principal crystal axes, $\mathrm{Cr}$ ions are numbered as 1, 2, 3, 4; $\mathrm{R}$ ions are numbered as 5, 6, 7, 8. Green arrows denote the orientation of electric dipole moments in the vicinity of $\mathrm{Cr}^{3+}$ ions ordered by antiferroelectric $\boldsymbol{D}$ mode. 
Magnetic moments of the $d$-ions determined by the vectors $M_{i}(i=1-4)$ constitute 4 transition metal magnetic sublattices, and the magnetic moments of the $f$-ions determined by vectors $m_{i}(i=1-4)$ constitute 4 rare-earth magnetic sublattices. The combinations between magnetic moments of the $d$ - and the $f$-sublattices determine magnetic modes of the ' $d$ ' ions:

$$
\begin{aligned}
& F=M_{1}+M_{2}+M_{3}+M_{4}, A=M_{1}-M_{2}-M_{3}+M_{4} \\
& G=M_{1}-M_{2}+M_{3}-M_{4}, C=M_{1}+M_{2}-M_{3}-M_{4}
\end{aligned}
$$

and magnetic modes of the ' $f$ '- ions:

$$
\begin{array}{r}
f=m_{1}+m_{2}+m_{3}+m_{4}, a=m_{1}-m_{2}-m_{3}+m_{4} \\
g=m_{1}-m_{2}+m_{3}-m_{4}, c=m_{1}+m_{2}-m_{3}-m_{4}
\end{array}
$$

Neutron diffraction measurements showed that $\mathrm{RCrO}_{3}$ exhibits one of three G-type AFM configurations with weak magnetic components $\Gamma_{1}\left(A_{x}, G_{y}, C_{z}\right), \Gamma_{2}\left(F_{x}, C_{y}, G_{z}\right)$, and $\Gamma_{4}$ $\left(G_{x} A_{y} F_{z}\right)$.

In contrast to $\mathrm{BiFeO}_{3}$, the ferroelectric properties of $\mathrm{RCrO}_{3}$ are weak, and the Curie ordering temperatures are low $\left(\mathrm{T}_{\mathrm{C}} \sim 130-250 \mathrm{~K}\right)[55,56]$. However, as recent experiments have shown, during the polishing of samples in an electric field, an electric polarization of the order of $0.06 \mu \mathrm{C} / \mathrm{cm}^{2}$ is induced; moreover, the electric field affects the spin-reorientation phase transitions, which indicates the presence of magnetoelectric coupling in the material $[57,58]$.

As expected, the magnetoelectric behavior is owed to the crystallographic distortions, which are quite different from those in $\mathrm{BiFeO}_{3}$. In the case of $\mathrm{RCrO}_{3}$, the principal distortions are related to the antirotation of the $\mathrm{CrO} 6$ octahedrons around the [110] direction and the displacements of $\mathrm{R}$ ions from A-positions in the perovskite parent phase. Octahedron rotation is described by the axial vectors $\omega_{i}(i=1 \div 4)$ linked to $\mathrm{Cr}$ ions, and, as was shown in Ref. [10], they contribute to the axial order parameter.

$$
\Omega_{b}=\omega_{1}-\omega_{2}+\omega_{3}-\omega_{4}
$$

Polar order parameters related to the displacements of oxygen and rare-earth ions are also allowed. To compose the polar order parameters, we consider the electric dipole moments $\boldsymbol{d}_{i}=q \boldsymbol{r}_{i}$ emerging in the vicinity of the Cr ions, where $\boldsymbol{r}_{q}=\sum_{i} q_{i} \boldsymbol{r}_{q i} / \sum_{i} q_{i}$ is the position of the electric dipole charge center, $q_{i}$ are the signed magnitudes of the charges, and $\boldsymbol{r}_{q i}$ are the radius vectors of the charges in the local reference frame. For the perovskite-like compounds,

$$
\boldsymbol{r}_{q}=\frac{\left(+\frac{3}{8} e\right) \cdot \sum_{i=1}^{8} \boldsymbol{r}_{R}+\left(-\frac{2}{2} e\right) \cdot \sum_{i=1}^{6} \boldsymbol{r}_{O}}{\left|8 \cdot\left(+\frac{3}{8} e\right)+6 \cdot\left(-\frac{2}{2} e\right)\right|} \quad \boldsymbol{r}=(x, y, z)
$$

where $e$ is the elementary charge, $\boldsymbol{r}_{R}$ are the radius vectors of the rare-earth ions, and $\boldsymbol{r}_{O}$ are the radius vectors of the oxygen ions measured from the $\mathrm{Cr}^{3+}$ ion.

As in the case of magnetic moments (7), (8), electric dipoles $d_{i}=q \boldsymbol{r}_{i}$ constitute 4 ferroelectric sublattices, and the combinations between dipole moments determine ferroelectric modes reads.

$$
\begin{gathered}
P=d_{1}+d_{2}+d_{3}+d_{4} \\
Q_{2}=d_{1}-d_{2}-d_{3}+d_{4} \\
Q_{3}=d_{1}-d_{2}+d_{3}-d_{4} \\
D=d_{1}+d_{2}-d_{3}-d_{4}
\end{gathered}
$$

The arrangement of the dipole moments in $\mathrm{RCrO}_{3}$ is shown in Figure 1; as can be seen, they form an antiferroelectric structure ordered according to the $\boldsymbol{D}$-mode. Direct calculation of the dipole moments shows that the value of $D$ is maximum, and the values of $P, Q_{2,3}$ are negligible. 
The transformation properties of the order parameters introduced above can be found using the irreducible representations of the space symmetry group of the $\mathrm{RCrO}_{3}$ compounds given in Table 2.

Table 2. Irreducible representations of the Pnma symmetry group.

\begin{tabular}{cccccccc}
\hline$\Gamma_{\boldsymbol{i}}$ & $\overline{\mathbf{1}}$ & $\mathbf{2}_{\mathbf{x}}$ & $\mathbf{2}_{\mathbf{y}}$ & $\mathbf{2}_{\mathbf{z}}$ & \multicolumn{2}{c}{ Magnetic OPs, Magnetic Field } & Structural OPs, Electric Field \\
\hline$\Gamma_{1}$ & 1 & 1 & 1 & 1 & $A_{z}, G_{x}, C_{y}$ & $4 \mathrm{c}$ & $\Omega_{b x}$ \\
\hline$\Gamma_{2}$ & 1 & 1 & -1 & -1 & $F_{z}, G_{y}, C_{x}, H_{z}$ & $f_{y}, c_{x}$ & $\Omega_{b y}$ \\
\hline$\Gamma_{3}$ & 1 & -1 & 1 & -1 & $F_{x}, A_{y}, C_{z}, H_{x}$ & $f_{x}, c_{z}$ & $\Omega_{b z}$ \\
\hline$\Gamma_{4}$ & 1 & -1 & -1 & 1 & $F_{y}, A_{x}, G_{z}, H_{y}$ & $f_{y}$ & $Q_{2 z}, Q_{3 x}, D_{z}$ \\
\hline$\Gamma_{5}$ & -1 & 1 & 1 & 1 & & $g_{z}, a_{x}$ & $P_{z}, E_{z}, Q_{3 y}$ \\
\hline$\Gamma_{6}$ & -1 & 1 & -1 & -1 & & $a_{y}$ & $P_{y}, Q_{2 x}, E_{y}, D_{x}$ \\
\hline$\Gamma_{7}$ & -1 & -1 & 1 & -1 & & $a_{z}, g_{x}$ & $P_{x}, Q_{2 y}, E_{x}, D_{y}$ \\
\hline$\Gamma_{8}$ & -1 & -1 & -1 & 1 & $g_{y}$ & \\
\hline
\end{tabular}

Knowledge of their transformation properties makes it possible to understand how crystallographic distortions manifest themselves in magnetic and ferroelectric orderings. As in the previous subsection, classification of the components of order parameters according to the irreducible representations of the Pnma symmetry group allows us to determine exchange-coupled magnetic structures, the possible electrostatically coupled dipole structures, to compose the invariant combination of order parameters contributing to the thermodynamic potential and to calculate the magnetoelectric coupling coefficient.

$$
\begin{gathered}
P_{x}=\left(\alpha_{x y x} a_{y}+\alpha_{x z y x} g_{z} G_{y} G_{x}+\alpha_{x x y x} a_{x} G_{y} G_{x}\right) H_{x}+\left(\alpha_{x x y} a_{x}+\alpha_{x x y y} f_{x} G_{y} G_{x}+\alpha_{x z y y} c_{z} G_{y} G_{x}+\alpha_{x z y} g_{z}\right) H_{y}+\ldots \\
F_{x}=\left(\alpha_{x y x}^{*} a_{y}+\alpha_{x z y x}^{*} g_{z} G_{y} G_{x}+\alpha_{x x y x}^{*} a_{x} G_{y} G_{x}\right) E_{x}+\left(\alpha_{x x y}^{*} a_{x}+\alpha_{x x y y}^{*} f_{x} G_{y} G_{x}+\alpha_{x z y y}^{*} c_{z} G_{y} G_{x}+\alpha_{x z y}^{*} g_{z}\right) E_{y}+\ldots
\end{gathered}
$$

The magnetoelectric coefficient in terms of the components of $G$ and $g$ vectors is written as follows.

$$
\alpha_{i j}^{R C r O_{3}}=\left|\begin{array}{lll}
a_{1} \boldsymbol{G}_{x}\left(\boldsymbol{g}_{z} \boldsymbol{G}_{y}-\boldsymbol{g}_{y} \boldsymbol{G}_{z}\right) & a_{2} \boldsymbol{g}_{z} & a_{3} \boldsymbol{g}_{x} \\
a_{2} \boldsymbol{g}_{z} & a_{1} \boldsymbol{G}_{x}\left(\boldsymbol{g}_{z} \boldsymbol{G}_{y}-\boldsymbol{g}_{y} \boldsymbol{G}_{z}\right) & a_{4} \boldsymbol{g}_{y} \\
a_{4} \boldsymbol{g}_{y} & a_{3} \boldsymbol{g}_{x} & a_{2} \boldsymbol{g}_{z}
\end{array}\right|
$$

At the end of this section, we draw the reader's attention to the fact that the presented analysis applies to rare-earth orthoferrites $\mathrm{RFeO}_{3} \cdot \mathrm{RFeO}_{3}$ compounds crystallize in the same space symmetry group Pnma, and several of these crystals exhibit nonlinear magnetoelectric responses $[23,25,59]$. The magnetoelectric tensor (12) can also be used to describe their magnetoelectric properties.

\subsection{Ruddlesden-Popper Structures}

Ruddlesden-Popper (RP) structures are attracting considerable attention due to the recently discovered high-temperature ferroelectric properties and the prospects for their potential implementation as multiferroics at room temperature [11]. The presence of ferroelectric properties, the so-called hybrid improper ferroelectricity (HIF), was theoretically predicted in double-layered perovskite compounds $\mathrm{Ca}_{3} \mathrm{Mn}_{2} \mathrm{O}_{7}$ and $\mathrm{Ca}_{3} \mathrm{Ti}_{2} \mathrm{O}_{7}$, and then detected experimentally in $\mathrm{Ca}_{3-\mathrm{x}} \mathrm{Sr}_{\mathrm{x}} \mathrm{Ti}_{2} \mathrm{O}_{7}$ single crystals [20], and in $\mathrm{Ca}_{3}\left(\mathrm{Ti}_{1-\mathrm{x}} \mathrm{Mn}_{\mathrm{x}}\right)_{2} \mathrm{O}_{7}$ ceramics [21].

In this section, we plan to focus on the RP manganite phases $\mathrm{Ca}_{3}\left(\mathrm{Ti}_{1-\mathrm{x}} \mathrm{Mn}_{\mathrm{x}}\right)_{2} \mathrm{O}_{7}$ as they contain magnetic Mn ions and, as shown in Ref. [21], are room-temperature HIF materials. In the limiting case $\mathrm{x}=1, \mathrm{Ca}_{3}\left(\mathrm{Ti}_{1-\mathrm{x}} \mathrm{Mn}_{\mathrm{x}}\right)_{2} \mathrm{O}_{7}$ transforms into the $\mathrm{RP}$ phase $\mathrm{Ca}_{3} \mathrm{Mn}_{2} \mathrm{O}_{7}$, 
which was thoroughly explored due to the colossal magnetoresistance effect found in the $(\mathrm{CaO})-\left(\mathrm{CaMnO}_{3}\right)_{\mathrm{n}}(\mathrm{n}=1,2,3, \infty) \mathrm{RP}$ structures in the 2000s [16-19]. The structures with $\mathrm{n}=1$ (2D-structure) and $\mathrm{n}=\infty$ (perovskite) are considered as the end members of the RP series, so the double-layered RP structures receive more attention.

The temperature of AFM ordering $\mathrm{T}_{\mathrm{N}}=115 \mathrm{~K}$ in $\mathrm{Ca}_{3} \mathrm{Mn}_{2} \mathrm{O}_{7}$ was first experimentally found in Ref. [16], where it was assumed that there is a G-type AFM order. A neutron diffraction study [19] revealed the dominant contribution of the G-type or C-type AFM phase with an AFM spin arrangement within the bi-layer plane. In that work, the possibility of the existence of a weak ferromagnetic (WFM) state in the RP phase $\mathrm{Ca}_{3} \mathrm{Mn}_{2} \mathrm{O}_{7}$ was also assumed. A further study on $\mathrm{Ca}_{3} \mathrm{Mn}_{2} \mathrm{O}_{7}$ showed the transition to the AFM state at the temperature $\mathrm{T}_{\mathrm{N}}=134 \mathrm{~K}$ and a signature of WFM below $100 \mathrm{~K}[60,61]$.

The crystal structure, structural phase transitions, and polar phases of $\mathrm{Ca}_{3} \mathrm{Ti}_{2} \mathrm{O}_{7}$ [62] and $\mathrm{Ca}_{3} \mathrm{Mn}_{2} \mathrm{O}_{7}[19,63]$ with double-layered RP structures, studied since 1998 [16], have now been proven [21]. Above room temperature (RT), the crystal structure of $\mathrm{Ca}_{3} \mathrm{Mn}_{2} \mathrm{O}_{7}$ is described by the tetragonal space group $I 4 / \mathrm{mmm}$, and at RT, by the space group $C m c 2_{1}$ [64]. The transition from the $I 4 / \mathrm{mmm}$ to $\mathrm{Cmc2} 2_{1}$ phase should occur through an intermediate phase, which can be either $\mathrm{Cmcm}$ or $\mathrm{Cmca}$, as follows from the first-principles calculations [46]. The transition into the ferroelectric phase is observed close to $T_{C}=280 \mathrm{~K}$, at which the transition into the $\mathrm{Cmc2}{ }_{1}$ phase occurs. As experimental measurements indicate that ferroelectric ordering in $\mathrm{Ca}_{3} \mathrm{Mn}_{2} \mathrm{O}_{7}$ occurs up to $\mathrm{RT}$, the existence of an intermediate ferroelectric state is expected here.

Structural transformations can arise due to the substitution of magnetic ions at the A-positions $\left(\mathrm{Ca}_{3-\mathrm{x}} \mathrm{La}_{\mathrm{x}} \mathrm{Mn}_{2} \mathrm{O}_{7}\right)$ or ferroelectric ions in the B-positions $\left(\mathrm{Ca}_{3}\left(\mathrm{Ti}_{1-\mathrm{x}} \mathrm{Mn}_{\mathrm{x}}\right)_{2} \mathrm{O}_{7}\right)$. The tetragonal-to-orthorhombic transition presumable through the phases $I 4 / \mathrm{mmm} \rightarrow$ $\mathrm{Fmmm} \rightarrow \mathrm{Cmcm} \rightarrow \mathrm{Cmc}_{2}$, occurring in the $200-300{ }^{\circ} \mathrm{C}$ range, was observed during the study of $\mathrm{Ca}_{3-x} \mathrm{La}_{x} \mathrm{Mn}_{2} \mathrm{O}_{7}$ structures [19,64]. Though the existence of $I 4 / \mathrm{mmm}$ and $\mathrm{Cmc2}_{1}$ phases is confirmed, the presence of 'intermediate' phases is still under discussion.

Thus, in further consideration, we can take the tetragonal I4/mmm structure $\left(\mathrm{Ca}_{3} \mathrm{Mn}_{2} \mathrm{O}_{7}\right)$ as the parent phase and apply the symmetry analysis, employed in the previous sections, to explore the magnetoelectric properties of the RP structures. According to the data of the synchrotron X-ray diffraction study [19], the Mn ions occupy the 4e positions (in the Wyckoff notation) in the I4/ $\mathrm{mmm}$ phase, which indicates the presence of 4 magnetic sublattices $\mu_{i}(i=1 \div 4)$ with magnetic moments of the same magnitude. Possible combinations of the vectors $\mu_{i}$ give us the basic magnetic vectors.

$$
\begin{aligned}
& F=\mu_{1}+\mu_{2}+\mu_{3}+\mu_{4} \\
& A=\mu_{1}-\mu_{2}-\mu_{3}+\mu_{4} \\
& G=\mu_{1}-\mu_{2}+\mu_{3}-\mu_{4} \\
& C=\mu_{1}+\mu_{2}-\mu_{3}-\mu_{4}
\end{aligned}
$$

The unit cell of $\mathrm{Ca}_{3} \mathrm{Mn}_{2} \mathrm{O}_{7}$ shown in Figure 2 contains 2 formula units $\mathrm{Ca}_{3} \mathrm{Mn}_{2} \mathrm{O}_{7}$. The positions of the symmetry elements $I, C_{2}^{z}, C_{4}^{z}, C_{2}^{y}$ (generators of $I 4 / \mathrm{mmm}$ space group) in the unit cell are depicted in Figure $2 \mathrm{~b}$ in accordance with Figure 2a. Note that the local symmetry elements of the $4 \mathrm{e}\{4 \mathrm{~mm}\}$ positions, which are the $4_{z}$ axis (principal crystal axis) and 4 planes of symmetry $m$ passing through this axis, remain even for magnetic ions, occupying the 4 e positions.

In accordance with Figure 2, it is easy to compose permutation transformations of the ions at the 4 e positions (Table 3 ) and permutation transformations of basis vectors (Table 4). 


\section{$I 4 / \mathrm{mmm}^{17} \quad D_{4 h}^{17}$}

No. 139

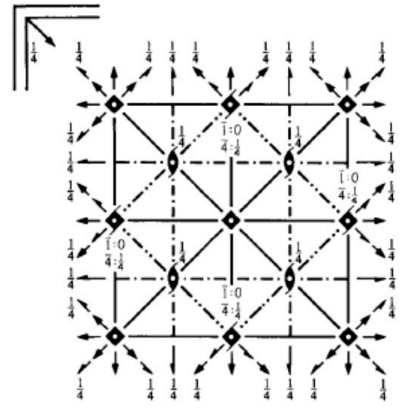

(a)

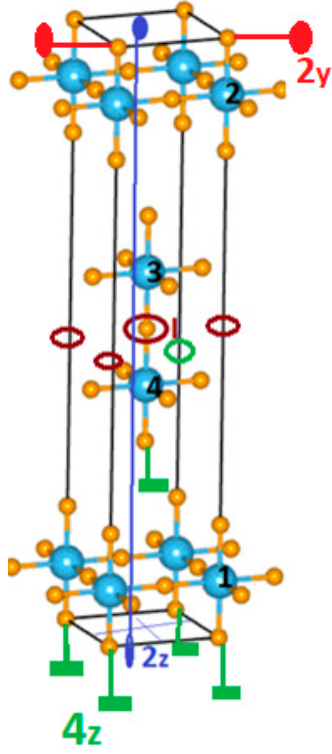

(b)

Figure 2. (a) $\mathrm{I} 4 / \mathrm{mmm}$ space symmetry group elements; (b) $\mathrm{Ca}_{3} \mathrm{Mn}_{2} \mathrm{O}_{7}$ unit cell, the numbering of magnetic ions is presented, the arrangement of the symmetry elements is also shown-the axes of symmetry $4_{z}, 2_{z}, 2_{y}$ and the centers of inversion $I$.

Table 3. Permutation transformations ions in positions $4 \mathrm{e}\{4 \mathrm{~mm}\}$.

\begin{tabular}{ccccc}
\hline $\mathbf{G}_{\mathbf{F}}$ & $\mathbf{1}$ & $\mathbf{2}$ & $\mathbf{3}$ & $\mathbf{4}$ \\
\hline$\overline{1}$ & 2 & 1 & 4 & 3 \\
\hline $4_{\mathrm{z}}$ & 1 & 2 & 3 & 4 \\
\hline $2_{\mathrm{z}}$ & 3 & 4 & 1 & 2 \\
\hline $2_{\mathrm{y}}$ & 2 & 1 & 4 & 3 \\
\hline
\end{tabular}

Table 4. Permutation transformations of basis vectors.

\begin{tabular}{ccccc}
\hline $\mathbf{G}_{\mathbf{F}}$ & $\mathbf{F}$ & $\mathbf{A}$ & $\mathbf{G}$ & $\mathbf{C}$ \\
\hline$\overline{1}$ & $\mathbf{F}$ & $-\mathbf{A}$ & $-\mathbf{G}$ & $\mathbf{C}$ \\
\hline $4_{\mathrm{z}}$ & $\mathbf{F}$ & $\mathbf{A}$ & $\mathbf{G}$ & $\mathbf{C}$ \\
\hline $2_{\mathrm{z}}$ & $\mathbf{F}$ & $-\mathbf{A}$ & $\mathbf{G}$ & $-\mathbf{C}$ \\
\hline $2_{\mathrm{y}}$ & $\mathbf{F}$ & $-\mathbf{A}$ & $-\mathbf{G}$ & $\mathbf{C}$ \\
\hline
\end{tabular}

Using Table 4, we obtain a cipher (Turov indices) for AFM vectors, which explains how the symmetry elements transform magnetic sublattices into each other.

$$
\begin{aligned}
& A: 4_{z}(+) 2_{z}^{1}(-) 2_{y}(-) \overline{1}(-) \\
& G: 4_{z}(+) 2_{z}^{1}(+) 2_{y}(-) \overline{1}(-) \\
& C: 4_{z}(+) 2_{z}^{1}(-) 2_{y}(+) \overline{1}(+)
\end{aligned}
$$

In consistence with the data of experimental studies [16,19], we assume the G-type of AFM ordering. In this case, the values of AFM vectors $A$ and $C$ are taken to be sufficiently small and we can restrict our consideration with two magnetic order parameters instead of 4. Thus, we leave the ferromagnetic vector $\boldsymbol{M}=\boldsymbol{F}$ and the antiferromagnetic vector $\boldsymbol{L}=\boldsymbol{G}$ as the basic magnetic parameters. Then, we classify them together with the polarization vector according to the irreducible representation of the space symmetry group $D_{4 h}^{17}$ (Table 5). The 
first line of Table 5 contains generators of the $D_{4 h}^{17}$ group $G=\left\{E, I, C_{2}^{y}, C_{2}^{z}, C_{4}^{z+}\right\}$, where the last column contains basic magnetic and ferroelectric functions.

Table 5. Irreducible representations of the $I 4 / \mathrm{mmm}$ symmetry group and basic functions.

\begin{tabular}{|c|c|c|c|c|c|c|}
\hline$\Gamma_{i}$ & $E$ & $2 C_{4 z}$ & $C_{2 z}$ & $2 C_{2 y}$ & $\overline{\mathbf{1}}$ & Basic Vectors $F, G, P$ \\
\hline$\Gamma_{1}$ & 1 & 1 & 1 & 1 & 1 & \\
\hline$\Gamma_{2}$ & 1 & 1 & 1 & -1 & 1 & $F_{z}$ \\
\hline$\Gamma_{3}$ & 1 & -1 & 1 & -1 & 1 & \\
\hline$\Gamma_{4}$ & 1 & -1 & 1 & 1 & 1 & \\
\hline$\Gamma_{5}$ & $\left.\begin{array}{ll}1 & 0 \\
0 & 1\end{array}\right)$ & $\left.\begin{array}{cc}0 & 1 \\
-1 & 0\end{array}\right)$ & $\left.\begin{array}{cc}-1 & 0 \\
0 & -1\end{array}\right)$ & $\left(\begin{array}{cc}-1 & 0 \\
0 & 1\end{array}\right)$ & $\left(\begin{array}{ll}1 & 0 \\
0 & 1\end{array}\right)$ & $\left(\begin{array}{l}F_{x} \\
F_{y}\end{array}\right)$ \\
\hline$\Gamma_{6}$ & 1 & 1 & 1 & 1 & -1 & $G_{z}$ \\
\hline$\Gamma_{7}$ & 1 & 1 & 1 & -1 & -1 & $P_{z}$ \\
\hline$\Gamma_{8}$ & 1 & -1 & 1 & -1 & -1 & \\
\hline$\Gamma_{9}$ & 1 & -1 & 1 & 1 & -1 & \\
\hline$\Gamma_{10}$ & $\left.\begin{array}{ll}1 & 0 \\
0 & 1\end{array}\right)$ & $\left.\begin{array}{cc}0 & 1 \\
-1 & 0\end{array}\right)$ & $\left.\begin{array}{cc}-1 & 0 \\
0 & -1\end{array}\right)$ & $\left(\begin{array}{cc}-1 & 0 \\
0 & 1\end{array}\right)$ & $\left.\begin{array}{cc}-1 & 0 \\
0 & -1\end{array}\right)$ & $\left(\begin{array}{l}P_{x} \\
P_{y}\end{array}\right)$ \\
\hline$\Gamma_{10}^{\prime}$ & $\left.\begin{array}{ll}1 & 0 \\
0 & 1\end{array}\right)$ & $\left.\begin{array}{cc}0 & 1 \\
-1 & 0\end{array}\right)$ & $\left.\begin{array}{cc}-1 & 0 \\
0 & -1\end{array}\right)$ & $\left(\begin{array}{cc}1 & 0 \\
0 & -1\end{array}\right)$ & $\left.\begin{array}{cc}-1 & 0 \\
0 & -1\end{array}\right)$ & $\left(\begin{array}{l}G_{x} \\
G_{y}\end{array}\right)$ \\
\hline
\end{tabular}

The decomposition of the basic functions into irreducible representations of the symmetry group $14 / \mathrm{mmm}$ allows one to obtain information on the properties of a system. It is seen that the ferromagnetic and antiferromagnetic vectors transform according to the different IRs. In this case the transformation of $\boldsymbol{F}$ and $\boldsymbol{L}$ into each other can occur due to external factors, such as the electric field or strain. The combinations $F_{z}\left(P_{x} G_{x}+P_{y} G_{y}\right), G_{z}\left(F_{x} P_{x}+F_{y} P_{y}\right), P_{z}\left(F_{x} G_{x}+F_{y} P_{z} G_{y}\right), F_{z} P_{z} G_{z}$, transforming on $\Gamma_{1}$, are invariants and have an impact on magnetoelectric energy.

$$
\Phi_{m e}=\gamma_{2} F_{z}\left(P_{x} G_{x}+P_{y} G_{y}\right)+\gamma_{3} G_{z}\left(F_{x} P_{x}+F_{y} P_{y}\right)+\gamma_{4} P_{z}\left(F_{x} G_{x}+F_{y} G_{y}\right)+\gamma_{5} F_{z} P_{z} G_{z}
$$

where

$$
\gamma_{2}=\gamma_{z x x}=\gamma_{z y y}, \gamma_{3}=\gamma_{x x z}=\gamma_{y y z}, \gamma_{4}=\gamma_{x z x}=\gamma_{y z y}, \gamma_{5}=\gamma_{z z z}
$$

Using Table 5 we calculate the magnetoelectric coupling tensor.

$$
\alpha_{i j}^{R-P}=\left|\begin{array}{lcc}
\gamma_{3} G_{z} & 0 & \gamma_{2} G_{x} \\
0 & \gamma_{3} G_{z} & \gamma_{2} G_{y} \\
\gamma_{4} G_{x} & \gamma_{4} G_{y} & \gamma_{5} G_{z}
\end{array}\right|
$$

As seen from Equation (16) it linearly depends on the components of antiferromagnetic vector $G$. This finding allows us to conclude that the linear magnetoelectric effect is allowed by the symmetry of the RP structures.

\section{Results and Conclusions}

To summarize, we applied symmetry analysis to the magnetic and magnetoelectric properties of multiferroics (MFs) with a perovskite structure. One of the reasons for choosing perovskite-based MFs as the object of this study is related to the structural instability of the initial perovskite phase, which can lead to a variety of structures with different symmetry. This allows us to demonstrate how crystallographic distortions of various types, even if they are small enough, significantly alter the magnetic and ferroelectric orderings and their couplings. As the typical examples, we considered (i) the family of high-temperature multiferroics $\mathrm{Bi}_{\mathrm{x}} \mathrm{R}_{1-\mathrm{x}} \mathrm{FeO}_{3}$ recognizable by their magnetoelectric proper- 
ties; (ii) the rare-earth orthochromites $\mathrm{RCrO}_{3}$, promising candidates for MFs, compounds with well-known magnetic structures; and (iii) the RP structures containing magnetic cations, such as $\mathrm{Ca}_{3}\left(\mathrm{Ti}_{1-\mathrm{x}} \mathrm{Mn}_{\mathrm{x}}\right)_{2} \mathrm{O}_{7}$, novel high-temperature MFs, the ferroelectric and magnetic properties of which are still being researched. These structures crystallize into trigonal, orthorhombic, and tetragonal syngonies, respectively, which allows us to trace the relationship between magnetoelectric properties and the symmetry of a structure, and to consider impacts given by crystallographic distortions.

The direct contribution of the distortion into electric polarization and weak ferromagnetism was considered, using $\mathrm{BiFeO}_{3}$ as an example. We also analyzed the influence of structural transformation of the $\mathrm{Bi}_{\mathrm{x}} \mathrm{R}_{1-\mathrm{x}} \mathrm{FeO}_{3}$ family on their magnetoelectric properties. Calculations showed that the linear magnetoelectric effect, suppressed by the spin-modulated structure in pure $\mathrm{BiFeO}_{3}$, becomes allowed due to the symmetry of the new phase in $\mathrm{Bi}_{x} \mathrm{R}_{1-x} \mathrm{FeO}_{3}$, where it is attributed mainly to rare earth ( $\mathrm{R}$ ) ions.

In the case of the rare-earth orthochromites $\mathrm{RCrO}_{3}$, it was shown that the ferroelectric and magnetoelectric properties are due to crystallographic distortions. The displacements of oxygen ions from their positions in the initial perovskite phase results in the emergence of electric dipole moments in the vicinity of the $\mathrm{Cr}$ ions, which are coupled with the magnetic moments of the $\mathrm{Cr}$ ions. The distortive, ferroelectric, and magnetic order parameters were classified according to irreducible representations of the Pnma symmetry group of $\mathrm{RCrO}_{3}$, which allows the invariant combinations to be composed between these parameters.

For the first time, a similar symmetry consideration was implemented in the analysis of the Ruddlesden-Popper structures. Taking into account the local symmetry of magnetic ions in the RP unit cell, we introduced the relevant magnetic order parameters and classified them according to the irreducible representation of the $14 / \mathrm{mmm}$ symmetry group, which describes the tetragonal symmetry of the RP structures. Analysis exemplified on the $\mathrm{Ca}_{3}\left(\mathrm{Ti}_{1-x} \mathrm{Mn}_{\mathrm{x}}\right)_{2} \mathrm{O}_{7}$ compounds demonstrated the possibility of realizing MEE in the RP phases containing magnetically active cations and allowed the estimation of the magnetoelectric contribution to the thermodynamic potential.

In conclusion, this research allowed us to compare the magnetoelectric effects for different crystal systems of perovskites and thus design a more meaningful organization of the desired experiments.

Author Contributions: Conceptualization, A.Z., Z.G. and X.C.; methodology, K.Z.; formal analysis, Z.G.; writing-review and editing, Z.G., A.Z., K.Z. and X.C. All authors have read and agreed to the published version of the manuscript.

Funding: Z.G. acknowledges support from the State assignment for the implementation of scientific research by laboratories (Order MN-8/1356 of 09/20/2021), Russian Foundation for Basic Research under grant No. 19-52-80024. A.Z. and K.Z. acknowledge the Russian Foundation for Basic Research under grant No. 19-52-80024. X.C. acknowledges the National Natural Science Foundation of China under Grant No. 51961145105.

Institutional Review Board Statement: Not applicable.

Informed Consent Statement: Not applicable.

Conflicts of Interest: The authors declare no conflict of interest.

\section{References}

1. Manipatruni, S.; Nikonov, D.E.; Lin, C.-C.; Gosavi, T.A.; Liu, H.; Prasad, B.; Huang, Y.-L.; Bonturim, E.; Ramesh, R.; Young, I.A. Scalable Energy-Efficient Magnetoelectric Spin-Orbit Logic. Nature 2019, 565, 35-42. [CrossRef]

2. Dubrovin, R.M.; Alyabyeva, L.N.; Siverin, N.V.; Gorshunov, B.P.; Novikova, N.N.; Boldyrev, K.N.; Pisarev, R.V. Incipient multiferroicity in Pnma fluoroperovskite $\mathrm{NaMnF}_{3}$. Phys. Rev. B 2020, 101, 180403. [CrossRef]

3. Liu, H.; Yang, X. A brief review on perovskite multiferroics. Ferroelectrics 2017, 507, 69-85. [CrossRef]

4. Ke, X.; Birol, T.; Misra, R.; Lee, J.-H.; Kirby, B.; Schlom, D.G.; Fennie, C.J.; Freeland, J.W. Structural control of magnetic anisotropy in a strain-driven multiferroic $\mathrm{EuTiO}_{3}$ thin film. Phys. Rev. B 2013, 88, 094434. [CrossRef]

5. Catalan, G.; Scott, J.F. Physics and Applications of Bismuth Ferrite. Adv. Mater. 2009, 21, 2463-2485. [CrossRef] 
6. Lin, P.-T.; Li, X.; Zhang, L.; Yin, J.-H.; Cheng, X.-W.; Wang, Z.-H.; Wu, Y.-C.; Wu, G.-H. La-doped BiFeO ${ }_{3}$ : Synthesis and multiferroic property study. Chin. Phys. B 2014, 23, 047701. [CrossRef]

7. Savosta, M.M.; Novák, P.; Maryško, M.; Jirák, Z.; Hejtmánek, J.; Englich, J.; Kohout, J.; Martin, C.; Raveau, B. Coexistence of antiferromagnetism and ferromagnetism in Ca1-xPrxMnO3 (x< 0.1)manganites. Phys. Rev. B 2000, 62, 9532-9537. [CrossRef]

8. Zvezdin, A.K.; Mukhin, A.A. Magnetoelectric interactions and phase transitions in a new class of multiferroics with improper electric polarization. JETP Lett. 2008, 88, 505-510. [CrossRef]

9. Shikin, A.M.; Estyunin, D.A.; Zaitsev, N.L.; Glazkova, D.; Klimovskikh, I.I.; Filnov, S.O.; Rybkin, A.G.; Schwier, E.F.; Kumar, S.; Kimura, A.; et al. Sample-dependent Dirac-point gap in MnBi2Te4 and its response to applied surface charge: A combined photoemission and ab initio study. Phys. Rev. B 2021, 104, 115168. [CrossRef]

10. Zvezdin, A.K.; Gareeva, Z.V.; Chen, X.M. Multiferroic Order Parameters in Rhombic Antiferromagnets RCrO 3 . J. Phys. Condens. Matter 2021, 33, 385801. [CrossRef]

11. Zhang, B.H.; Liu, X.Q.; Chen, X.M. Review of experimental progress of hybrid improper ferroelectricity in layered perovskite oxides. J. Phys. D Appl. Phys. 2021, 55, 113001. [CrossRef]

12. Evans, H.A.; Mao, L.; Seshadri, R.; Cheetham, A.K. Layered Double Perovskites. Annu. Rev. Mater. Sci. 2021, 51, 351-380. [CrossRef]

13. Ruddlesden, S.N.; Popper, P. The compound $\mathrm{Sr}_{3} \mathrm{Ti}_{2} \mathrm{O}_{7}$ and its structure. Acta Crystallogr. 1958, 11, 54-55. [CrossRef]

14. Ding, P.; Li, W.; Zhao, H.; Wu, C.; Zhao, L.; Dong, B.; Wang, S. Review on Ruddlesden-Popper perovskites as cathode for solid oxide fuel cells. J. Phys. Mater. 2021, 4, 022002. [CrossRef]

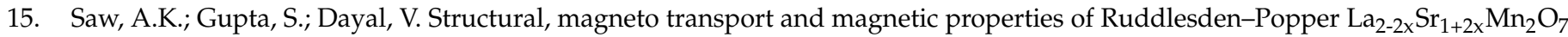
$(0.42 \leq \mathrm{x} \leq 0.52)$ layered manganites. AIP Adv. 2021, 11, 025331. [CrossRef]

16. Battle, P.D.; Rosseinsky, M.J. Synthesis, structure, and magnetic properties of $\mathrm{n}=2$ Ruddlesden-Popper manganates. Curr. Opin. Solid State Mater. Sci. 1999, 4, 163-170. [CrossRef]

17. Fawcett, I.D.; Sunstrom; Greenblatt, M.; Croft, M.; Ramanujachary, K.V. Structure, Magnetism, and Properties of Ruddlesden-Popper Calcium Manganates Prepared from Citrate Gels. Chem. Mater. 1998, 10, 3643-3651. [CrossRef]

18. Harris, A.B. Symmetry analysis for the Ruddlesden-Popper systems $\mathrm{Ca}_{3} \mathrm{Mn}_{2} \mathrm{O}_{7}$ and $\mathrm{Ca}_{3} \mathrm{Ti}_{2} \mathrm{O}_{7}$. Phys. Rev. B 2011, 84, 064116 . [CrossRef]

19. Lobanov, M.; Greenblatt, M.; Caspi, E.A.N.; Jorgensen, J.D.; Sheptyakov, D.; Toby, B.; Botez, C.E.; Stephens, P.W. Crystal and magnetic structure of the $\mathrm{Ca}_{3} \mathrm{Mn}_{2} \mathrm{O}_{7}$ Ruddlesden-Popper phase: Neutron and synchrotron x-ray diffraction study. J. Phys. Condens. Matter 2004, 16, 5339-5348. [CrossRef]

20. Oh, Y.S.; Luo, X.; Huang, F.-T.; Wang, Y.; Cheong, S.-W. Experimental demonstration of hybrid improper ferroelectricity and the presence of abundant charged walls in $(\mathrm{Ca}, \mathrm{Sr})_{3} \mathrm{Ti}_{2} \mathrm{O}_{7}$ crystals. Nat. Mater. 2015, 14, 407-413. [CrossRef] [PubMed]

21. Liu, X.Q.; Wu, J.W.; Shi, X.X.; Zhao, H.J.; Zhou, H.Y.; Qiu, R.H.; Zhang, W.Q.; Chen, X.M. Hybrid improper ferroelectricity in Ruddlesden-Popper $\mathrm{Ca}_{3}(\mathrm{Ti}, \mathrm{Mn})_{2} \mathrm{O}_{7}$ ceramics. Appl. Phys. Lett. 2015, 106, 202903. [CrossRef]

22. Glazer, A.M. The classification of tilted octahedra in perovskites. Acta Crystallogr. Sect. B Struct. Crystallogr. Cryst. Chem. 1972, 28, 3384-3392. [CrossRef]

23. Senn, M.S.; Bristowe, N. A group-theoretical approach to enumerating magnetoelectric and multiferroic couplings in perovskites Acta Crystallogr. Sect. A Found. Adv. 2018, 74, 308-321. [CrossRef] [PubMed]

24. Mulder, A.T.; Benedek, N.A.; Rondinelli, J.M.; Fennie, C.J. Turning ABO3Antiferroelectrics into Ferroelectrics: Design Rules for Practical Rotation-Driven Ferroelectricity in Double Perovskites and $\mathrm{A}_{3} \mathrm{~B}_{2} \mathrm{O}_{7}$ Ruddlesden-Popper Compounds. Adv. Funct. Mater. 2013, 23, 4810-4820. [CrossRef]

25. Bousquet, E.; Cano, A. Non-collinear magnetism in multiferroic perovskites. J. Phys. Condens. Matter 2016, 28, 123001. [CrossRef] [PubMed]

26. Narayanan, N.; Graham, P.J.; Rovillain, P.; O’Brien, J.; Bertinshaw, J.; Yick, S.; Hester, J.; Maljuk, A.; Souptel, D.; Büchner, B.; et al Reduced Crystal Symmetry as Origin of the Ferroelectric Polarization within the Incommensurate Magnetic Phase of $\mathrm{TbMn}_{2} \mathrm{O}_{5}$. arXiv 2021, arXiv:2109.05164.

27. Mato, J.M.P.; Ribeiro, J.L.; Petricek, V.; Aroyo, M.I. Magnetic superspace groups and symmetry constraints in incommensurate magnetic phases. J. Phys. Condens. Matter 2012, 24, 163201. [CrossRef]

28. Hatch, D.M.; Stokes, H.T. INVARIANTS: Program for obtaining a list of invariant polynomials of the order-parameter components associated with irreducible representations of a space group. J. Appl. Crystallogr. 2003, 36, 951-952. [CrossRef]

29. (International Tables for Crystallography) Introduction to the Properties of Tensors. Available online: https: / / onlinelibrary.wiley. com/iucr/itc/Da/ch1o1v0001/ (accessed on 27 December 2021).

30. Gabbasova, Z.; Kuz'min, M.; Zvezdin, A.; Dubenko, I.; Murashov, V.; Rakov, D.; Krynetsky, I. Bi $i_{-x} \mathrm{R}_{\mathrm{x}} \mathrm{FeO}_{3}(\mathrm{R}=\mathrm{rare}$ earth): A family of novel magnetoelectrics. Phys. Lett. A 1991, 158, 491-498. [CrossRef]

31. Palkar, V.R.; John, J.; Pinto, R. Observation of saturated polarization and dielectric anomaly in magnetoelectric $\mathrm{BiFeO}_{3}$ thin films. Appl. Phys. Lett. 2002, 80, 1628-1630. [CrossRef]

32. Lebeugle, D.; Colson, D.; Forget, A.; Viret, M.; Bonville, P.; Marucco, J.F.; Fusil, S. Room-temperature coexistence of large electric polarization and magnetic order in $\mathrm{BiFeO}_{3}$ single crystals. Phys. Rev. B 2007, 76, 024116. [CrossRef]

33. Lebeugle, D.; Colson, D.; Forget, A.; Viret, M. Very large spontaneous electric polarization in $\mathrm{BiFeO}_{3}$ single crystals at room temperature and its evolution under cycling fields. Appl. Phys. Lett. 2007, 91, 022907. [CrossRef] 
34. Teague, J.R.; Gerson, R.; James, W. Dielectric hysteresis in single crystal BiFeO 3 . Solid State Commun. 1970, 8, 1073-1074. [CrossRef]

35. Wang, Y.P.; Yuan, G.L.; Chen, X.Y.; Liu, J.-M.; Liu, Z.G. Electrical and magnetic properties of single-phased and highly resistive ferroelectromagnet $\mathrm{BiFeO}_{3}$ ceramic. J. Phys. D: Appl. Phys. 2006, 39, 2019-2023. [CrossRef]

36. Li, J.; Wang, J.; Wuttig, M.; Ramesh, R.; Wang, N.; Ruette, B.; Pyatakov, A.P.; Zvezdin, A.K.; Viehland, D. Dramatically enhanced polarization in (001), (101), and (111) $\mathrm{BiFeO}_{3}$ thin films due to epitiaxial-induced transitions. Appl. Phys. Lett. 2004, 84, 5261-5263. [CrossRef]

37. Dixit, H.; Beekman, C.; Schlepütz, C.M.; Siemons, W.; Yang, Y.; Senabulya, N.; Clarke, R.; Chi, M.; Christen, H.M.; Cooper, V.R. Understanding Strain-Induced Phase Transformations in $\mathrm{BiFeO}_{3}$ Thin Films. Adv. Sci. 2015, 2, 1500041. [CrossRef] [PubMed]

38. Sando, D.; Agbelele, A.; Rahmedov, D.; Liu, J.; Rovillain, P.; Toulouse, C.; Infante, I.; Pyatakov, A.P.; Fusil, S.; Jacquet, E.; et al. Crafting the magnonic and spintronic response of $\mathrm{BiFeO}_{3}$ films by epitaxial strain. Nat. Mater. 2013, 12, 641-646. [CrossRef]

39. Resta, R.; Vanderbilt, D. Theory of Polarization: A Modern Approach. In Physics of Ferroelectrics: A Modern Perspective; Topics in Applied Physics; Springer: Berlin/Heidelberg, Germany, 2007; pp. 31-68. [CrossRef]

40. Spaldin, N.A. A beginner's guide to the modern theory of polarization. J. Solid State Chem. 2012, 195, 2-10. [CrossRef]

41. Gareeva, Z.; Diéguez, O.; Iñiguez, J.; Zvezdin, A.K. Complex domain walls in BiFeO3. Phys. Rev. B 2015, 91, 060404. [CrossRef]

42. Zvezdin, A.K.; Pyatakov, A.P. On the Problem of Coexistence of the Weak Ferromagnetism and the Spin Flexoelectricity in Multiferroic Bismuth Ferrite. EPL Europhys. Lett. 2012, 99, 57003. [CrossRef]

43. Fishman, R.S.; Rõõm, T.; de Sousa, R. Normal modes of a spin cycloid or helix. Phys. Rev. B 2019, 99, 064414. [CrossRef]

44. Sosnowska, I.; Neumaier, T.P.; Steichele, E. Spiral magnetic ordering in bismuth ferrite. J. Phys. C Solid State Phys. 1982, 15, 4835-4846. [CrossRef]

45. Kadomtseva, A.M.; Zvezdin, A.K.; Popov, Y.F.; Pyatakov, A.P.; Vorob'Ev, G.P. Space-time parity violation and magnetoelectric interactions in antiferromagnets. JETP Lett. 2004, 79, 571-581. [CrossRef]

46. Kadomtseva, A.M.; Popov, Y.F.; Pyatakov, A.P.; Vorob'Ev, G.P.; Zvezdin, A.K.; Viehland, D. Phase transitions in multiferroic BiFeO3crystals, thin-layers, and ceramics: Enduring potential for a single phase, room-temperature magnetoelectric 'holy grail'. Phase Transitions 2006, 79, 1019-1042. [CrossRef]

47. Popov, Y.F.; Kadomtseva, A.M.; Vorob'Ev, G.P.; Zvezdin, A.K. Discovery of the linear magnetoelectric effect in magnetic ferroelectric $\mathrm{BiFeO}_{3}$ in a strong magnetic field. Ferroelectrics 1994, 162, 135-140. [CrossRef]

48. Pyatakov, A.P.; Zvezdin, A.K. Magnetoelectric and multiferroic media. Phys.-Uspekhi 2012, 55, 557-581. [CrossRef]

49. Murashov, V.A.; Rakov, D.N.; Ehkonomov, N.A. Quadratic Magnetoelectric Effect in Monocrystalline (Bi,La)FeO 3 . Fiz. Tverd. Tela 1990, 32, 2156-2158.

50. Sosnowska, I.; Przeniosło, R.; Fischer, P.; Murashov, V. Neutron diffraction studies of the crystal and magnetic structures of $\mathrm{BiFeO}_{3}$ and $\mathrm{Bi}_{0.93} \mathrm{La}_{0.07} \mathrm{FeO}_{3}$. J. Magn. Magn. Mater. 1996, 160, 384-385. [CrossRef]

51. Chen, J.; Liu, L.; Zhu, X.L.; Gareeva, Z.V.; Zvezdin, A.K.; Chen, X.M. The involvement of Pna21 phase in the multiferroic characteristics of La/Lu co-substituted $\mathrm{BiFeO}_{3}$ ceramics. Appl. Phys. Lett. 2021, 119, 112901. [CrossRef]

52. Belov, K.P.; Zvezdin, A.K.; Kadomtseva, A.M.; Levitin, R.Z. Spin-reorientation transitions in rare-earth magnets. Sov. Phys. Uspekhi 1976, 19, 574. [CrossRef]

53. Turov, E.A. Can the magnetoelectric effect coexist with weak piezomagnetism and ferromagnetism? Phys.-Uspekhi 1994, 37, 303-310. [CrossRef]

54. Izyumov, Y.A.; Naish, V.E. Symmetry Analysis in Neutron Diffraction Studies of Magnetic Structures: 1. A Phase Transition Concept to Describe Magnetic Structures in Crystals. J. Magn. Magn. Mater. 1979, 12, 239-248. [CrossRef]

55. Tassel, C.; Goto, Y.; Kuno, Y.; Hester, J.; Green, M.; Kobayashi, Y.; Kageyama, H. Direct Synthesis of Chromium Perovskite Oxyhydride with a High Magnetic-Transition Temperature. Angew. Chem. 2014, 126, 10545-10548. [CrossRef]

56. Singh, K.D.; Singh, F.; Choudhary, R.J.; Kumar, R. Consequences of R3+ Cationic Radii on the Dielectric and Magnetic Behavior of $\mathrm{RCrO}_{3}$ Perovskites. Appl. Phys. A 2020, 126, 148. [CrossRef]

57. Sanina, V.A.; Khannanov, B.K.; Golovenchits, E.I.; Shcheglov, M.P. Electric Polarization in ErCrO3 Induced by Restricted Polar Domains. Phys. Solid State 2019, 61, 370-378. [CrossRef]

58. Rajeswaran, B.; Khomskii, D.I.; Zvezdin, A.K.; Rao, C.N.R.; Sundaresan, A. Field-Induced Polar Order at the N $\backslash$ 'eel Temperature of Chromium in Rare-Earth Orthochromites: Interplay of Rare-Earth and Cr Magnetism. Phys. Rev. B 2012, 86, 214409. [CrossRef]

59. Tokunaga, Y.; Furukawa, N.; Sakai, H.; Taguchi, Y.; Arima, T.; Tokura, Y. Composite Domain Walls in a Multiferroic Perovskite Ferrite. Nat. Mater. 2009, 8, 558-562. [CrossRef]

60. Sahlot, P.; Jana, A.; Awasthi, A.M. Exchange Bias in Multiferroic Ca $\mathrm{Mn}_{2} \mathrm{O}_{7}$ Effected by Dzyaloshinskii-Moriya Interaction. AIP Conf. Proc. 2018, 1942, 130009. [CrossRef]

61. Jung, W.-H. Weak Ferromagnetism of $\mathrm{n}=2$ Ruddlesden: Popper $\mathrm{Ca}_{3} \mathrm{Mn}_{2} \mathrm{O}_{7}$ System. J. Mater. Sci. Lett. 2000, 19, 2037-2038 [CrossRef]

62. Elcombe, M.M.; Kisi, E.H.; Hawkins, K.D.; White, T.J.; Goodman, P.; Matheson, S. Structure Determinations for Ca $\mathrm{Ci}_{2} \mathrm{O}_{7}$, $\mathrm{Ca}_{4} \mathrm{Ti}_{3} \mathrm{O}_{10}, \mathrm{Ca}_{3.6} \mathrm{Sr}_{0.4} \mathrm{Ti}_{3} \mathrm{O}_{10}$ and a Refinement of $\mathrm{Sr}_{3} \mathrm{Ti}_{2} \mathrm{O}_{7}$. Acta Crystallogr. B 1991, 47, 305-314. [CrossRef]

63. Guiblin, N.; Grebille, D.; Leligny, H.; Martin, C. $\mathrm{Ca}_{3} \mathrm{Mn}_{2} \mathrm{O}_{7}$. Acta Crystallogr. C 2002, 58, i3-i5. [CrossRef] [PubMed]

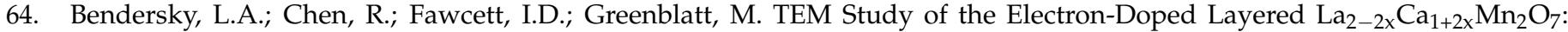
Orthorhombic Phase in the 0.8. J. Solid State Chem. 2001, 157, 309-323. [CrossRef] 